\title{
Fatigue and physical disability in patients with multiple sclerosis: a structural equation modeling approach
}

\author{
Yvonne Bol • Annelien A. Duits • Richel Lousberg • \\ Raymond M. M. Hupperts • Michelle H. P. Lacroix • \\ Frans R. J. Verhey · Johan W. S. Vlaeyen
}

Received: August 28, 2009/Accepted: May 4, 2010/Published online: May 28, 2010

(C) The Author(s) 2010. This article is published with open access at Springerlink.com

\begin{abstract}
Although fatigue is one of the most common and disabling symptoms in patients with multiple sclerosis (MS), its pathogenesis is still poorly understood and it is difficult to treat. The aim of the current study was to test the assumptions of a cognitive-behavioral model that explains fatigue and physical disability in MS patients, by comparing this approach with a more traditional biomedical approach. Structural equation modeling was applied to a sample of 262 MS patients. Neither the
\end{abstract}

Y. Bol $(\bowtie)$

Department of Medical Psychology and Psychiatry, Orbis Medical Centre, PO Box 5500, 6130 MB Sittard,

The Netherlands

e-mail: Y.bol@maastrichtuniversity.nl

Y. Bol · A. A. Duits · R. Lousberg · R. M. M. Hupperts ·

F. R. J. Verhey

School for Mental Health and Neuroscience, Maastricht

University Medical Centre, Maastricht, The Netherlands

A. A. Duits · R. Lousberg · F. R. J. Verhey

Department of Psychiatry and Psychology, Maastricht University

Medical Centre, Maastricht, The Netherlands

R. M. M. Hupperts

Department of Neurology, Orbis Medical Centre, Sittard, The Netherlands

M. H. P. Lacroix

Faculty of Health and Technology, Zuyd University, Heerlen,

The Netherlands

J. W. S. Vlaeyen

Department of Clinical Psychological Science, Maastricht

University, Maastricht, The Netherlands

J. W. S. Vlaeyen

Department of Psychology, University of Leuven, Leuven,

Belgium cognitive-behavioral, nor the biomedical model showed an adequate fit of our data. The modification indices supported an integration of both models, which showed a better fit than those of the separate models. This final model, is notable for at least three features: (1) fatigue is associated with depression and physical disability, (2) physical disability is associated with disease severity and fatigue-related fear and avoidance behavior, and (3) catastrophic interpretations about fatigue, fueled by depression, mediated the relationship between fatigue and fatiguerelated fear and avoidance behavior. Our results suggest that an integrated approach, including the modification of catastrophic thoughts about fatigue, would be beneficial in the treatment of fatigue in MS patients.

Keywords Multiple sclerosis · Fatigue - Catastrophizing · Physical disability - Cognitive-behavioral model .

Integrated approach

\section{Introduction}

Multiple sclerosis (MS) is a chronic inflammatory demyelinating disease of the central nervous system with an unknown pathogenesis and an unpredictable course. MS is considered to be one of the most common neurological disorders affecting young adults (Compston and Coles 2008) The clinical picture of MS is diverse and includes physical and neuropsychiatric symptoms (Feinstein 1999). Up to $90 \%$ of patients with MS complain of fatigue (Branas et al. 2000). Fatigue in MS patients is more severe and disabling compared to fatigue in healthy controls and several other somatic populations (De Ridder et al. 2004; Krupp 2006; Krupp et al. 1988, 1989; Penner et al. 2007; Trojan et al. 2007), and over two-thirds of MS patients 
characterize it as their most troubling symptom (Branas et al. 2000). The pathogenesis of MS-related fatigue is still poorly understood and treatment options are limited (Bol et al. 2009; Kos et al. 2008). In clinical practice, fatigue is often treated by both pharmacological and non-pharmacological interventions, such as exercise therapy and energy conservation strategies (Kos et al. 2008). However, the empirical evidence for effectiveness of these interventions is limited (Pucci et al. 2007; Rietberg et al. 2005; Solari et al. 2003; Steultjens et al. 2003).

From a traditional biomedical perspective, MS-related fatigue is considered to be a primary symptom of MS that is directly related to the severity of the disease. Although there is some evidence for underlying pathophysiological mechanisms, including inflammation, demyelinisation, axonal loss, and neuroendocrine dysregulation, these variables appear to explain only a small part of the variance of both MS-related fatigue and MS-related disability (Bol et al. 2009; Kos et al. 2008). More recently, a cognitive-behavioral approach has been proposed which suggests that fatigue is not perpetuated or worsened by the severity of the disease or associated symptoms, but by the individual's interpretation of these symptoms (Skerrett and Moss-Morris 2006; Van Kessel and Moss-Morris 2006). Empirical evidence for such an approach has been found in cases of chronic fatigue syndrome (Deary et al. 2007; Nijs et al. 2004; Silver et al. 2002), cancer survivors (Donovan et al. 2007), and chronic pain (Arntz and Claassens 2004; Leeuw et al. 2007).

One of the key variables in recent cognitive-behavioral approaches of symptom reporting is the way information is interpreted. For example, catastrophic interpretations are characterized by exaggerated negative rumination, amplification of the symptoms, and helplessness (Leeuw et al. 2007; Sullivan et al. 1995), which are all associated with negative emotions. In particular, catastrophizing is associated with specific, symptom-related, fear which in turn incites avoidance behavior and in the long term contributes to disability (Andrea et al. 2004; Jacobsen et al. 2004; Nijs et al. 2004; Silver et al. 2002). There is also evidence that MS patients who catastrophize about their fatigue and who attribute their fatigue to their illness are more likely to focus their attention on signs of fatigue and to interpret the consequences of fatigue in terms of physical damage (Skerrett and Moss-Morris 2006). A second feature of a cognitivebehavioral approach is its focus on functioning in the presence of persistent symptoms such as pain and fatigue. There is evidence that the functional disability levels are more strongly influenced by the emotional responses to the symptoms than the symptoms themselves (e.g. Crombez et al. 1999).

The hypothesis of the cognitive-behavioral model was that patients who interpret their fatigue catastrophically, report more fatigue-related fear and avoidance, and hence are more physically disabled, depressed and fatigued (Skerrett and Moss-Morris 2006; Van Kessel and MossMorris 2006). In contrast, the biomedical model assigns a more prominent role to disease severity and depression in predicting the level of fatigue (Amato et al. 2001; Janardhan and Bakshi 2002; Merkelbach et al. 2002) and considers catastrophizing about fatigue, and fatigue-related fear and avoidance, to be consequences rather than precursors of physical disability.

In present study we compared a model based on a cognitive-behavioral approach with that based on a more traditional, biomedical approach. Given the merits of both approaches, a comparison of both approaches is not only interesting theoretically, but may also provide elements for a better care of patients with MS-related fatigue and disability. Using structural equation modeling (SEM), we compared the fit indices of our data according to both theoretical models. In line with evidence in other populations (Deary et al. 2007; Donovan et al. 2007; Leeuw et al. 2007; Nijs et al. 2004; Silver et al. 2002), we expected to find that the cognitive-behavioral model would show a better fit than the traditional biomedical model.

The results of this study may have clinical implications. The first, and so far only, randomized clinical trial of cognitive-behavioral therapy (CBT) in MS patients appeared to be more effective in reducing self-reported fatigue than relaxation therapy (Van Kessel et al. 2008). Not only fatigue, but also fatigue-related disability and depression showed a significant decrease. These results are in favor of a cognitive-behavioral approach to treat fatigue, but the theoretical assumptions of CBT were not formally tested yet. The focus of the present study is to test the underlying mechanisms of CBT. Insight into how the various relevant factors may affect one another, can help us to develop more responsive interventions to treat MS-related fatigue and disability.

\section{Method}

\section{Participants}

Participants were recruited from the hospital databases of the Departments of Neurology of the Maastricht University Medical Centre and Atrium Medical Centre Parkstad Heerlen. A total of 404 patients aged 18 years and older, and diagnosed with clinically definite MS (McDonald et al. 2001), were eligible for inclusion. To preserve patient confidentiality, the initial letters were sent to these $404 \mathrm{MS}$ patients by their treating neurologist. In total 294 patients were willing to participate and contacted the researchers (73\% response rate) and all these 294 patients were screened by telephone to verify eligibility. Four patients 
were excluded because they had problems understanding the questionnaires. Furthermore, ten patients who had suffered from a (recent) exacerbation and were taking corticosteroids were included 4 weeks later when their condition was stabilized. In total 290 patients were sent a patient information letter, a consent form and questionnaires. Only 20 patients failed to return these forms, leaving the total sample at 270 (93\% response rate). The medical ethics committee of both the Maastricht University Medical Centre and Atrium Medical Centre Parkstad Heerlen approved the study and patients gave their informed consent. Patients did not receive any financial compensation for their participation.

\section{Measures}

Basic demographic information, including age, gender, marital status, level of education, employment status, a history of psychiatric and somatic diseases and use of psychopharmaca was obtained with the help of a demographic inventory filled out by the MS patients. The level of education was assessed by classifying formal schooling in three groups: those with at most primary education (low level of education); those with junior vocational training (average level of education); and those with senior vocational or academic training (high level of education). Medical data, including disease severity, disease course, disease duration, and MS medication, were collected from the hospital databases.

\section{Disease severity}

The Expanded Disability Status Scale (EDSS) (Kurtzke 1983), which was assessed by an experienced neurologist familiar with EDSS recording (RH), provided a measure of disease severity. The EDSS is divided into eight functioning systems (pyramidal, cerebellar, brainstem, mental, bowel and bladder, visual-optic, sensory, and other). Impairment in each system is graded separately by means of neurological examination. EDSS scores range from 0 to 10 , with 0 being normal neurological examination and 10 being death due to MS. If there was no recent ( $>3$ months) EDSS score available, the treating neurologist was consulted for a new assessment.

All participants completed the Dutch versions of the following questionnaires:

The Abbreviated Fatigue Questionnaire (AFQ) (Alberts et al. 1997) was used to measure physical fatigue. The AFQ is a valid, reliable, and easy-to-use instrument (Alberts et al. 1997), that consists of four items ('I feel tired', 'I tire easily', 'I feel fit' and 'I feel physically exhausted'), which are rated on a seven-point Likert scale with scoring alter- natives ranging from "yes, that is true" to "no, that is not true". After inverting the items 1, 2 and 4, a total score was calculated (range 4-28), with higher scores indicating higher severity of physical fatigue.

Catastrophizing about fatigue was measured with the Fatigue Catastrophizing Scale (FCS), which is an adapted version of the Pain Catastrophizing Scale (PCS) (Crombez and Vlaeyen 1996; Sullivan et al. 1995). The PCS is a 13-item questionnaire that measures the frequency of catastrophizing thoughts reported by patients about the pain they experience. Psychometric properties of the PCS appeared adequate (Crombez et al. 1998; Van Damme et al. 2000). We adapted the PCS by replacing the word 'pain' by the word 'fatigue' in all items. Scoring alternatives ranged from 'strongly disagree' to 'strongly agree'. Three MSrelated items were added ("When I am tired, this is a signal there is something wrong in my brain", "When I am tired, this is a warning for physical decline", "When I am tired, this is a sign that my MS is getting worse"). The FCS consists of 16 items, with scores ranging from 0 to 64 and with higher scores indicating higher intensity.

The fatigue version of the Tampa Scale for Kinesiophobia (TSK-F) (Silver et al. 2002) was used to measure fatigue-related fear and avoidance behavior. The TSK-F is an adapted version of the original 17-item TSK for chronic pain (Miller et al. 1991; Vlaeyen et al. 1995). The TSK is a valid and reliable instrument (Vlaeyen et al. 1995; Roelofs et al. 2007), and measures the extent to which patients believe that pain associated with physical activity is a sign of physical damage, and the extent to which they avoid physical activity because of these beliefs. To make this questionnaire adequate for fatigue, the word 'pain' was replaced by the word 'fatigue' and the items were adjusted, e.g., "I am afraid that I might make my symptoms worse if I exercise", instead of "I'm afraid that I might injure myself if I exercise". Each item is provided with a four-point Likert scale with scoring alternatives ranging from 'not at all' to 'all the time' (with scores ranging from 17 to 68 ).

Depression was measured with the subscale depression of the Hospital Anxiety and Depression Scale (HADS) (Zigmond and Snaith 1983). The HADS is specially designed to screen physically ill patients for anxiety and depression and does not include somatic symptoms. The depression scale consists of seven items, with depression scores ranging from 0 to 21 and with higher scores indicating higher intensity. Scores over seven indicate that MS patients are likely to be depressed (Honarmand and Feinstein 2009). Reliability and validity are adequate for several clinical populations, including MS (Bjelland et al. 2002; Honarmand and Feinstein 2009; Spinhoven et al. 1997).

The physical dimension of the Short Form Health Survey (SF-36) (Aaronson et al. 1998) was used to measure physical disability. The physical dimension includes four 
subscales, viz., physical functioning, role limitations due to physical health problems, bodily pain, and general health. The standardized scores (ranging form 0 to 100) of each subscale were added to lower scores that indicated a higher level of physical disability. The SF-36 was found to have adequate psychometric properties (Aaronson et al. 1998).

\section{Statistical analyses}

Data analysis was performed using SPSS 16.0.1 for Windows (SPSS Inc., Chicago, IL). Data were checked for missing values, normality and outliers. Of the 270 returned questionnaires, eight cases were excluded because too many values were missing ( $\geq 25 \%$ of items of questionnaire missing or $\geq 50 \%$ if a questionnaire consisted of four items). For all other cases $(n=26)$ all missing values were random and imputed by inserting the mean of the remaining non-missing items of the subscale. Any variables that were significantly skewed (skewness $<-1$ or $>1$ ) were transformed appropriately before parametric testing. Descriptive statistics were used to describe the sample. Cronbach's alpha was used to test reliability of all questionnaires. Pearson-correlations were used to analyze relations between all variables. The models were tested and modified with the help of the computer program AMOS 16.0.1 (Arbuckle 2007). We tested a measurement model with directly observed variables in which the error terms associated with the observed variables were left free to be estimated and also were assumed to be uncorrelated with each other. In line with the recommendations of Byrne (2001), model fit was assessed using several fit indices, viz., chi-square statistic $\left(\chi^{2}\right)$, goodness-of-fit index (GFI), adjusted goodness-of-fit index (AGFI), root mean square error of approximation (RMSEA), comparative fit index (CFI) and the consistent akaike information criteria (CAIC) respectively.

According to guidelines for SEM, the data were considered to fit the model if the $\chi^{2}$, which is a statistical test of lack of-fit resulting from overidentifying restrictions placed in a model, was not significant. $\chi^{2}$ is the most frequently used goodness-of-fit index. A statistically significant $\chi^{2}$ indicates that a significant amount of observed covariance between items remains unexplained by the model, while a non-significant $\chi^{2}$ implies a good fit of the model to the data. However, this index is sensitive to sample size, which is a disadvantage (Schumacker and Lomax 2004). In a small sample, a poor fit may result in a non-significant $\chi^{2}$, indicating a good fit. In a large sample, a good fit may

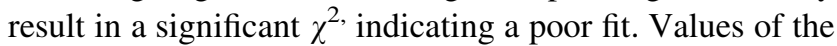
GFI and the AGFI that assess the extent to which a model provides a better fit compared to no model at all, should be high, respectively, above 0.95 and 0.85 . The RMSEA was taken into account as a measure of discrepancy per degree of freedom. RMSEA estimates lack of-fit in a model compared to a perfect model, and should therefore be small. RMSEA values up to 0.05 indicate a close fit, whereas values ranging between 0.08 and 0.10 indicate a mediocre fit, and those greater than 0.10 indicate a poor fit. The CFI represents the proportionate improvement in model fit by comparing the target model with a baseline model, usually a null model in which all the observed variables are uncorrelated; CFI values larger than 0.95 indicate an adequate fit. The CAIC is used to compare two or more not-nested models, with smaller values representing a better fit.

\section{Results}

Patient sample

In total 262 Caucasian outpatients (69 male, 193 female) were included in this study. Their mean age was 47.6 years $(\mathrm{SD}=11.7$, range $21-80)$. Most of them $(n=$ 136) had a relapsing remitting disease course, while 67 patients had a secondary progressive, and 59 patients a primary progressive course. The average EDSS score was $4.0(\mathrm{SD}=2.2$, range $0-8)$, which reflects a moderate disease severity. See Table 1 for a summary of all patient characteristics.

Table 2 presents means, standard deviations, ranges, Cronbach's alphas for all variables under study, as well as their intercorrelations (Pearson). All questionnaires had excellent internal consistency as measured by Cronbach's alpha, ranging from 0.82 to 0.91 . Although all intercorrelations were statistically significant $(P<0.01)$, the strongest one was found between catastrophizing about fatigue and fatigue-related fear and avoidance behavior. Higher levels of catastrophizing about fatigue were related to higher levels of fatigue-related fear-avoidance behavior $(r=0.64, P<0.001)$. The lowest intercorrelation was found between disease severity and catastrophizing about fatigue $(r=0.17, P<0.01)$.

Structural equation modeling analyses

An overview of all goodness-of-fit indices is displayed in Table 3. Figure 1 depicts the SEM results for the cognitive-behavioral model (model 1). None of the goodness-offit indices satisfied the a priori criteria for a good model fit. Although all hypothesized relationships of the biomedical model (model 2, Fig. 2) were statistically significant, SEM analyses of this model also revealed an unacceptable fit. As such, neither of the initial models that were designed a priori fitted our data. 
Table 1 Patient characteristics $(n=262)$

\begin{tabular}{|c|c|c|}
\hline Gender ( $\%$ male/\% female) & $26 / 74$ & \\
\hline Age in years (mean, (SD)) & $47.6(11.7)$ & Range 21.1-79.9 \\
\hline Disease duration in years (mean, $(\mathrm{SD})$ ) & $8.6(7.9)$ & Range $0.1-53.7$ \\
\hline Disease course (\% RR, \% SP, \% PP) & $52 / 26 / 22$ & \\
\hline EDSS (mean, (SD)) & $4.0(2.2)$ & Range $0-8$ \\
\hline HADS-depression $(\%<8 / \% \geq 8)$ & $68 / 32$ & \\
\hline Use of disease modifying drugs ( $\%$ yes, $\%$ no) & $43.5 / 56.5$ & \\
\hline Interferon $(\%)$ & 18.3 & \\
\hline Glatiramer acetate $(\%)$ & 3.8 & \\
\hline Other $(\%)$ & 15.3 & \\
\hline Use of psychopharmaca (\% yes, \% no) & $26 / 74$ & \\
\hline Level of education ( $\%$ low, $\%$ average, $\%$ high) & $28 / 36 / 36$ & \\
\hline Marital status (\% partner/\% no partner) & $78 / 22$ & \\
\hline Employment status (\% working, $\%$ not working) & $30 / 70$ & \\
\hline
\end{tabular}

$R R$ Relapsing Remitting, SP Secondary Progressive, PP Primary Progressive, EDSS Expanded Disability Status Scale, HADS Hospital Anxiety and Depression Scale

Table 2 Means, standard deviations (SD), range, Cronbach's alpha's $(\alpha)$ and Pearson-correlations of all measures

\begin{tabular}{lcccccccc}
\hline & Mean (SD) & Range & $\alpha$ & 2 & 3 & 4 & 5 \\
\hline 1. Disease severity (EDSS) & $4.0(2.2)$ & $0-8$ & - & $0.27^{* *}$ & $0.17^{*}$ & $0.25^{* *}$ & $0.26^{* *}$ & $-0.58^{* *}$ \\
2. Fatigue (AFQ) & $19.8(6.6)$ & $4-28$ & 0.91 & - & $0.56^{* *}$ & $0.37^{* *}$ & $0.49^{* *}$ & $-0.60^{* *}$ \\
3. Catastrophizing about fatigue (FCS) & $20.4(15.1)$ & $0-64$ & 0.91 & - & - & $0.64^{* *}$ & $0.57^{* *}$ & $-0.57^{* *}$ \\
4. Fatigue-related fear and avoidance (TSK-F) & $37.9(9.4)$ & $17-61$ & 0.82 & - & - & - & $0.50^{* *}$ & $-0.56^{* *}$ \\
5. Depression (HADS-D) & $6.0(4.2)$ & $0-21$ & 0.83 & - & - & - & - & $-0.57^{* *}$ \\
6. Physical disability (SF-36) & $246.3(87.0)$ & $15-390$ & 0.91 & - & - & - & - & - \\
\hline
\end{tabular}

EDSS Expanded Disability Status Scale, $A F Q$ Abbreviated Fatigue Questionnaire, FCS Fatigue Catastrophizing Scale, TSK-F Fatigue Version of the Tampa Scale for Kinesiophobia, HADS Hospital Anxiety and Depression Scale, SF-36 Short Form Health Survey

$* P<0.01 ; * * P<0.001$

Table 3 Goodness-of-fit summary for the models tested

\begin{tabular}{|c|c|c|c|c|c|c|}
\hline & $\chi^{2}(d f)$ & RMSEA & GFI & AGFI & CFI & CAIC \\
\hline Model 1. Cognitive-behavioral model & $202.77(8)^{* *}$ & 0.305 & 0.814 & 0.512 & 0.709 & 288.16 \\
\hline Model 2. Biomedical model & $107.76(7)^{* *}$ & 0.235 & 0.902 & 0.706 & 0.85 & 199.71 \\
\hline \multicolumn{7}{|l|}{ Model 1a: added } \\
\hline Disease severity to physical disability & $102.15(7)^{* *}$ & 0.228 & 0.886 & 0.659 & 0.858 & 194.11 \\
\hline \multicolumn{7}{|l|}{ Model 1b: added } \\
\hline Physical disability to depression & $52.54(6)^{* *}$ & 0.172 & 0.94 & 0.789 & 0.931 & 151.06 \\
\hline \multicolumn{7}{|l|}{ Model 1c: added } \\
\hline Depression to catastrophizing & $25.93(5)^{* *}$ & 0.127 & 0.969 & 0.87 & 0.969 & 131.02 \\
\hline \multicolumn{7}{|l|}{ Model 1d: added } \\
\hline Disease severity to fear-avoidance & $14.86(4)^{*}$ & 0.102 & 0.982 & 0.905 & 0.984 & 126.52 \\
\hline \multicolumn{7}{|l|}{ Final model (model 3): deleted } \\
\hline Non-significant paths & $17.44(6)^{*}$ & 0.085 & 0.979 & 0.925 & 0.983 & 115.96 \\
\hline
\end{tabular}

GFI Goodness-of-Fit Index, AGFI Adjusted Goodness-of-Fit Index, RMSEA Root Mean Square Error of Approximation, CFI Comparative Fit Index, CAIC Consistent Akaike Information Criteria

$* P<0.01 ; * * P<0.001$ 


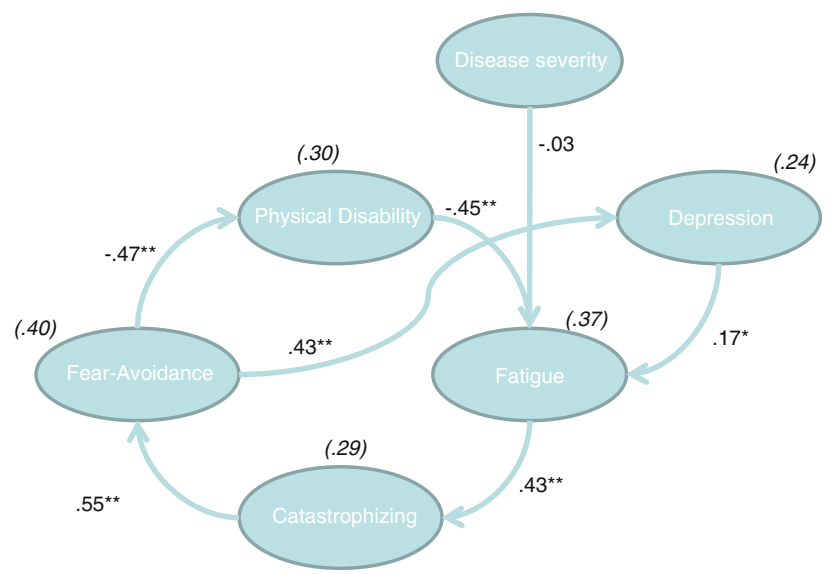

Fig. 1 Cognitive-behavioral model. Fear-Avoidance Fatigue-related fear and avoidance behavior, Catastrophizing catastrophizing about fatigue. Values shown are standardized regression coefficients. Explained variances are provided in parentheses. $* p<.01$; $* * p<.0 .001$

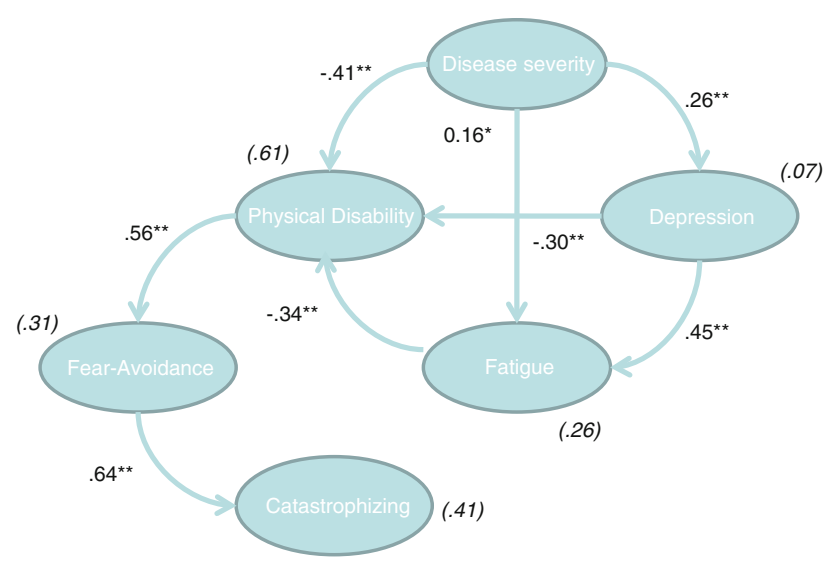

Fig. 2 Biomedical model. Fear-Avoidance Fatigue-related fear and avoidance behavior, Catastrophizing catastrophizing about fatigue. Values shown are standardized regression coefficients. Explained variances are provided in parentheses. $* p<.01$; ** $p<.0 .001$

Although SEM is generally used to test pre-specified conceptual models, it is often also applied in an exploratory way (Arbuckle 2007). Since there is empirical support for both cognitive-behavioral and biomedical factors in explaining MS-related fatigue, it was anticipated that the exploratory use of SEM, including factors of both models, would improve the overall understanding of fatigue and physical disability in MS. To explore whether a better fitting model existed, we adapted the cognitive-behavioral model by adding relationships suggested by the modification indices provided by the AMOS program (Byrne 2001; Schumacker and Lomax 2004). A modification index (MI) represents the value that $\chi^{2}$ is expected to decrease if such a relationship would be included. In this way new causal relationships that improve the model can be identified. Because SEM is a theoretically driven technique, we only

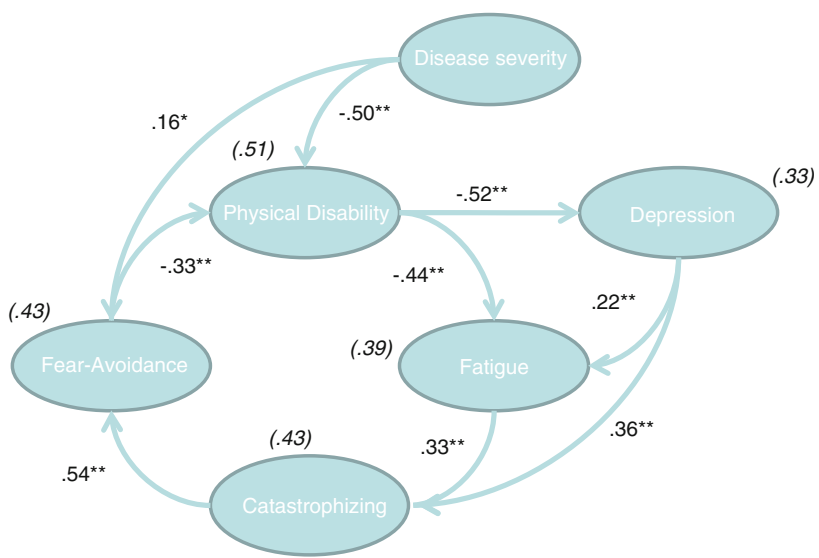

Fig. 3 Final model. Fear-Avoidance Fatigue-related fear and avoidance behavior, catastrophizing catastrophizing about fatigue. Values shown are standardized regression coefficients. Explained variances are provided in parentheses. $* p<.01 ; * * p<.0 .001$

added theoretically meaningful modifications. We subsequently added the relationships between disease severity and physical disability $(\mathrm{MI}=80.10)$; physical disability and depression ( $\mathrm{MI}=33.77)$; depression and catastrophizing about fatigue $(\mathrm{MI}=12.97)$; and disease severity and fatigue-related fear and avoidance behavior ( $\mathrm{MI}=$ 10.56). Finally, we successively deleted non-significant relationships in order to explain as much variance as possible with a minimum of variables (principle of maximum parsimony). We deleted two non-significant relationships, respectively, the path from disease severity to fatigue, and from fatigue-related fear and avoidance behavior to depression, and subsequently refitted each model. Although the RMSEA still showed a mediocre fit and the $\chi^{2}$ was still significant, the final model (see Table 3 and Fig. 3) produced the lowest CAIC value, indicating the best fit of all the models proposed.

\section{Discussion}

The aim of the current study was to test the assumptions of a cognitive-behavioral model by comparing these with those of a traditional biomedical model. In particular, we tested and explored the role of catastrophic (mis)interpretations on MS-related fatigue and physical disability. In contrast to the biomedical model, the cognitive-behavioral model's main assumption is that fatigue is not perpetuated or worsened by disease severity or associated symptoms, but by the patient's interpretation of these symptoms.

SEM revealed that neither of these models showed adequate fit of our data and the modification indices suggested an integrative approach. As such, the cognitivebehavioral model was subsequently modified by adding several theoretically meaningful relationships derived from 
the biomedical approach and deleting non-significant relationships. Although, the final model did not meet all preset fit criteria, it showed the best fit indices. The suboptimal fit of the final model could be due to the omittedvariable problem, which means that we failed to have included other relevant variables such as sleep problems, disease course, and medication use. Inclusion of such variables in future work may improve the fit indices. Even though some relevant variables may be lacking in the final model, its present structure is generally in line with that of the fear-avoidance model of chronic pain (Vlaeyen and Linton 2000; Leeuw et al. 2007) and with evidence for the joint contribution of biomedical and psychological factors in the prediction of pain and disability (Peters et al. 2005). The integrated model is notable for at least three main features: first, fatigue is associated with depression and physical disability. Second, physical disability is associated with disease severity and fatigue-related fear and avoidance behavior, and third, catastrophic interpretations about fatigue, -fueled by depression-, mediate the relationship between fatigue and fatigue-related fear and avoidance behavior.

Catastrophic interpretations of fatigue such as "When I am tired, this is a signal there is something wrong in my brain" and "When I am tired, this is a sign that my MS is getting worse" seemed to be a key factor and mediated the relationship between fatigue and fatigue-related fear and avoidance behavior. Hence, catastrophizing about fatigue may be an important target for cognitive-behavioral interventions during which dysfunctional fatigue-related thoughts can be challenged (e.g. Swain 2000). In this kind of cognitive treatment, patients are given the opportunity to test the credibility of their beliefs in catastrophic outcomes, and to correct these accordingly.

Furthermore, depression appeared to play an important role in our final model. Depression was a negative consequence of increased disability, and it directly contributed to fatigue and catastrophizing about fatigue. Depression is known to be the most common psychiatric disorder in MS, with an estimated prevalence ranging between 27 and 54\% (Minden et al. 1987; Minden and Schiffer 1990; Sadovnick et al. 1996; Schiffer et al. 1983). Therefore, we recommend the inclusion of depression in screening procedures and treatment for fatigued MS patients.

In contrast to the fear-avoidance model in chronic pain, disease severity played a prominent role in our final model. Disease severity indirectly contributed to fatigue, through fatigue-related fear and avoidance behavior, and physical disability. MS patients with higher levels of disease severity seem to experience more fear-related cognitions, and hence more physical disability. One of the reasons why disease severity in MS has a prominent role in prediction of fatigue and disability may be that the disease and the dis- ease-related symptoms of MS are much less ambiguous as compared other chronic illness conditions such as in patients with chronic pain and chronic fatigue syndrome.

While our results are in line with those of the first randomized clinical trial of cognitive-behavioral therapy (CBT) for fatigue in MS patients (Van Kessel et al. 2008), they also suggest that optimal treatment could be a combination of CBT and medical therapy. Given that most MS patients already receive medical treatment, more efforts should be directed towards the cognitive and behavioral aspects of fatigue. Since the CBT trial from Van Kessel e.a (2008), was rather non-specific and focusing - in only one session-on changing negative thoughts, our findings suggests that its effectiveness might be increased by better tailored CBT. Fatigued MS patients could benefit more if the target of CBT is on challenging idiosyncratic catastrophizing thoughts about fatigue and not negative thoughts in general. Such a treatment would be similar to exposurebased interventions for patients who report increasing painrelated fear (e.g. De Jong et al. 2005; Bailey et al. 2010).

There are several limitations to this study, which should be taken into account when interpreting the results and which may be addressed in future studies. First of all, the design is cross-sectional, making it impossible to draw firm conclusions about causal relationships between variables. Prospective and longitudinal studies are warranted to confirm causality. Secondly, because of our relatively small sample size, we were not able to split our sample in order to cross-validate our final model. Thirdly, we used postal questionnaires to collect our data. Although we had a favorable response rate for a postal questionnaire, we were not able to examine the differences between responders and non-responders. As a result, we cannot exclude the fact that a potential selection bias might have interfered with the present results. Fourthly, our measures for catastrophizing about fatigue (FCS), and fatigue-related fear and avoidance behavior (TSK-F), are adaptations of valid instruments. Although both measures show excellent internal consistency, their reliability and validity need to be established further. Finally, all data, including those on physical disability, were self-reported and therefore amenable to retrospective bias and social desirability effects. Furthermore, it is possible that strong associations between self-reported variables are the result of shared-method variance (Podsakoff et al. 2003). Future studies should also include more objective measures such as an accelerometer, which is a reliable and valid measure of daily activity levels (Bassett 2000; Ng and Kent-Braun 1997).

Despite the limitations described above, this study shows that not only severity of disease, but also cognitivebehavioral factors, such as catastrophizing about fatigue and fatigue-related fear and avoidance behavior, have to be considered when trying to explain fatigue and physical 
disability in MS patients. As such our results suggest that an integrated approach, would be beneficial in the treatment of fatigue in MS patients.

\section{Conflict of interest statement None.}

Open Access This article is distributed under the terms of the Creative Commons Attribution Noncommercial License which permits any noncommercial use, distribution, and reproduction in any medium, provided the original author(s) and source are credited.

\section{References}

Aaronson, N. K., Muller, M., Cohen, P. D., Essink-Bot, M. L., Fekkes, M., Sanderman, R., et al. (1998). Translation, validation, and norming of the Dutch language version of the SF-36 Health Survey in community and chronic disease populations. Journal of Clinical Epidemiology, 51(11), 1055-1068.

Alberts, M., Smets, E. M., Vercoulen, J. H., Garssen, B., \& Bleijenberg, G. (1997). 'Abbreviated fatigue questionnaire': A practical tool in the classification of fatigue. Nederlands Tijdschrift voor Geneeskunde, 141(31), 1526-1530.

Amato, M. P., Ponziani, G., Rossi, F., Liedl, C. L., Stefanile, C., \& Rossi, L. (2001). Quality of life in multiple sclerosis: The impact of depression, fatigue and disability. Multiple Sclerosis, 7(5), 340-344.

Andrea, H., Beurskens, A. J., Kant, I., Davey, G. C., Field, A. P., \& van Schayck, C. P. (2004). The relation between pathological worrying and fatigue in a working population. Journal of Psychosomatic Research, 57(4), 399-407.

Arbuckle, J. L. (2007). Amos 16.0 user's guide. Spring House: Amos Development Corporation.

Arntz, A., \& Claassens, L. (2004). The meaning of pain influences its experienced intensity. Pain, 109(1-2), 20-25.

Bailey, K. M., Carleton, R. N., Vlaeyen, J. W., \& Asmundson, G. J. (2010). Treatments addressing pain-related fear and anxiety in patients with chronic musculoskeletal pain: A preliminary review. Cognitive Behaviour Therapy, 39(1), 46-63.

Bassett, D. R., Jr. (2000). Validity and reliability issues in objective monitoring of physical activity. Research Quarterly for Exercise and Sport, 71(2), S30-S36.

Bjelland, I., Dahl, A. A., Haug, T. T., \& Neckelmann, D. (2002). The validity of the hospital anxiety and depression scale. An updated literature review. Journal of Psychosomatic Research, 52(2), 69-77.

Bol, Y., Duits, A. A., Hupperts, R. M., Vlaeyen, J. W., \& Verhey, F. R. (2009). The psychology of fatigue in patients with multiple sclerosis: A review. Journal of Psychosomatic Research, 66(1), 3-11.

Branas, P., Jordan, R., Fry-Smith, A., Burls, A., \& Hyde, C. (2000). Treatments for fatigue in multiple sclerosis: A rapid and systematic review. Health Technology Assessment, 4(27), 1-61.

Byrne, B. M. (2001). Structural equation modeling with AMOS. Basis concepts, applications and programming. New Jersey: Lawrence Erlbaum Associates.

Compston, A., \& Coles, A. (2008). Multiple sclerosis. Lancet, 372(9648), 1502-1517.

Crombez, G., Vervaet, L., Lysens, R., Baeyens, F., \& Eelen, P. (1998). Avoidance and confrontation of painful, back-straining movements in chronic back pain patients. Behavioral Modification, 22(1), 62-77.

Crombez, G., \& Vlaeyen, J. W. S. (1996). The Pain Catastrophizing Scale. Unpublished manuscript.
Crombez, G., Vlaeyen, J. W. S., Heuts, P. H. T. G., \& Lysens, R. (1999). Pain-related fear is more disabling than pain itself: Evidence on the role of pain-related fear in chronic back pain disability. Pain, 80(1-2), 329-339.

De Jong, J. R., Vlaeyen, J. W., Onghena, P., Cuypers, C., den Hollander, M., \& Ruijgrok, J. (2005). Reduction of pain-related fear in complex regional pain syndrome type I: The application of graded exposure in vivo. Pain, 116(3), 264-275.

De Ridder, D., Fournier, M., \& Bensing, J. (2004). Does optimism affect symptom report in chronic disease? What are its consequences for self-care behaviour and physical functioning? Journal of Psychosomatic Research, 56(3), 341-350.

Deary, V., Chalder, T., \& Sharpe, M. (2007). The cognitive behavioural model of medically unexplained symptoms: A theoretical and empirical review. Clinical Psychology Review, 27(7), 781-797.

Donovan, K. A., Small, B. J., Andrykowski, M. A., Munster, P., \& Jacobsen, P. B. (2007). Utility of a cognitive-behavioral model to predict fatigue following breast cancer treatment. Health Psychology, 26(4), 464-472.

Feinstein, A. (1999). The clinical neuropsychiatry of multiple sclerosis. Cambridge: University Press.

Honarmand, K., \& Feinstein, A. (2009). Validation of the hospital anxiety and depression scale for use with multiple sclerosis patients. Multiple Sclerosis, 15(12), 1518-1524.

Jacobsen, P. B., Andrykowski, M. A., Thors, C. L., Broeckel, J. A., Horton, J., Balducci, L., et al. (2004). Relationship of catastrophizing to fatigue among women receiving treatment for breast cancer. Journal of Consulting and Clinical Psychology, 72(2), 355-361.

Janardhan, V., \& Bakshi, R. (2002). Quality of life in patients with multiple sclerosis. The impact of fatigue and depression. Journal of the Neurological Sciences, 205(1), 51-58.

Kos, D., Kerckhofs, E., Nagels, G., D'Hooghe, M. B., \& Ilsbroukx, S. (2008). Origin of fatigue in multiple sclerosis: Review of the literature. Neurorehabilitation and Neural Repair, 22(1), 91-100.

Krupp, L. (2006). Fatigue is intrinsic to multiple sclerosis (MS) and is the most commonly reported symptom of the disease. Multiple Sclerosis, 12(4), 367-368.

Krupp, L. B., Alvarez, L. A., LaRocca, N. G., \& Scheinberg, L. C. (1988). Fatigue in multiple sclerosis. Archives of Neurology, 45(4), 435-437.

Krupp, L. B., LaRocca, N. G., Muir-Nash, J., \& Steinberg, A. D. (1989). The fatigue severity scale. Application to patients with multiple sclerosis and systemic lupus erythematosus. Archives of Neurology, 46(10), 1121-1123.

Kurtzke, J. F. (1983). Rating neurologic impairment in multiple sclerosis: An expanded disability status scale (EDSS). Neurology, 33(11), 1444-1452.

Leeuw, M., Goossens, M. E., Linton, S. J., Crombez, G., Boersma, K., \& Vlaeyen, J. W. (2007). The fear-avoidance model of musculoskeletal pain: Current state of scientific evidence. Journal of Behavioral Medicine, 30(1), 77-94.

McDonald, W. I., Compston, A., Edan, G., Goodkin, D., Hartung, H. P., Lublin, F. D., et al. (2001). Recommended diagnostic criteria for multiple sclerosis: Guidelines from the international panel on the diagnosis of multiple sclerosis. Annals of Neurology, 50(1), 121-127.

Merkelbach, S., Sittinger, H., \& Koenig, J. (2002). Is there a differential impact of fatigue and physical disability on quality of life in multiple sclerosis? Journal of Nervous and Mental Disease, 190(6), 388-393.

Miller, R. P., Kori, S. H., \& Todd, D. D. (1991). The Tampa scale. Unpublished Report.

Minden, S. L., Orav, J., \& Reich, P. (1987). Depression in multiple sclerosis. General Hospital Psychiatry, 9(6), 426-434. 
Minden, S. L., \& Schiffer, R. B. (1990). Affective disorders in multiple sclerosis. Review and recommendations for clinical research. Archives of Neurology, 47(1), 98-104.

Ng, A. V., \& Kent-Braun, J. A. (1997). Quantitation of lower physical activity in persons with multiple sclerosis. Medicine and Science in Sports and Exercise, 29(4), 517-523.

Nijs, J., De Meirleir, K., \& Duquet, W. (2004). Kinesiophobia in chronic fatigue syndrome: Assessment and associations with disability. Archives of Physical Medicine and Rehabilitation, 85(10), 1586-1592.

Penner, I. K., Bechtel, N., Raselli, C., Stocklin, M., Opwis, K., Kappos, L., et al. (2007). Fatigue in multiple sclerosis: Relation to depression, physical impairment, personality and action control. Multiple Sclerosis, 13(9), 1161-1167.

Peters, M. L., Vlaeyen, J. W., \& Weber, W. E. (2005). The joint contribution of physical pathology, pain-related fear and catastrophizing to chronic back pain disability. Pain, 113(1-2), 45-50.

Podsakoff, P. M., MacKenzie, S. B., Lee, J. Y., \& Podsakoff, N. P. (2003). Common method biases in behavioral research: A critical review of the literature and recommended remedies. Journal of Applied Psychology, 88(5), 879-903.

Pucci, E., Branas, P., D’Amico, R., Giuliani, G., Solari, A., \& Taus, C. (2007). Amantadine for fatigue in multiple sclerosis. Cochrane Database of Systematic Reviews, 1, CD002818.

Rietberg, M. B., Brooks, D., Uitdehaag, B. M., \& Kwakkel, G. (2005). Exercise therapy for multiple sclerosis. Cochrane Database of Systematic Reviews, 1, CD003980.

Roelofs, J., Sluiter, J. K., Frings-Dresen, M. H., Goossens, M., Thibault, P., Boersma, K., et al. (2007). Fear of movement and (re)injury in chronic musculoskeletal pain: Evidence for an invariant two-factor model of the Tampa scale for Kinesiophobia across pain diagnoses and Dutch, Swedish, and Canadian samples. Pain, 131(1-2), 181-190.

Sadovnick, A. D., Remick, R. A., Allen, J., Swartz, E., Yee, I. M., Eisen, K., et al. (1996). Depression and multiple sclerosis. Neurology, 46(3), 628-632.

Schiffer, R. B., Caine, E. D., Bamford, K. A., \& Levy, S. (1983). Depressive episodes in patients with multiple sclerosis. American Journal of Psychiatry, 140(11), 1498-1500.

Schumacker, R. E., \& Lomax, R. G. (2004). A beginner's guide to structural equation modeling. New Jersey: Lawrence Erlbaum Associates.

Silver, A., Haeney, M., Vijayadurai, P., Wilks, D., Pattrick, M., \& Main, C. J. (2002). The role of fear of physical movement and activity in chronic fatigue syndrome. Journal of Psychosomatic Research, 52(6), 485-493.
Skerrett, T. N., \& Moss-Morris, R. (2006). Fatigue and social impairment in multiple sclerosis: The role of patients' cognitive and behavioral responses to their symptoms. Journal of Psychosomatic Research, 61(5), 587-593.

Solari, A., Uitdehaag, B., Giuliani, G., Pucci, E., \& Taus, C. (2003). Aminopyridines for symptomatic treatment in multiple sclerosis. Cochrane Database of Systematic Reviews, 2, CD001330.

Spinhoven, P., Ormel, J., Sloekers, P. P., Kempen, G. I., Speckens, A. E., \& Van Hemert, A. M. (1997). A validation study of the hospital anxiety and depression scale (HADS) in different groups of Dutch subjects. Psychological Medicine, 27(2), 363-370.

Steultjens, E. M., Dekker, J., Bouter, L. M., Cardol, M., Van de Nes, J. C., \& Van den Ende, C. H. (2003). Occupational therapy for multiple sclerosis. Cochrane Database of Systematic Reviews, 3, CD003608.

Sullivan, M. J., Bishop, S. R., \& Pivik, J. (1995). The pain catastrophizing scale: Development and validation. Psychological Assessment, 7(4), 524-532.

Swain, M. G. (2000). Fatigue in chronic disease. Clinical Science, 99 , $1-8$.

Trojan, D., Arnold, D., Collet, J. P., Shapiro, S., Bar-Or, A., Robinson, A., et al. (2007). Fatigue in multiple sclerosis: Association with disease-related, behavioural and psychosocial factors. Multiple Sclerosis, 13(8), 985-995.

Van Damme, S., Crombez, G., Vlaeyen, J. W. S., Goubet, L., Van den Broeck, A., \& Houdenhove, B. (2000). De pain catastrophizing scale: Psychometrische karakteristieken en normering. Gedragstherapie, 33(3), 209-220.

Van Kessel, K., \& Moss-Morris, R. (2006). Understanding multiple sclerosis fatigue: A synthesis of biological and psychological factors. Journal of Psychosomatic Research, 61(5), 583-585.

Van Kessel, K., Moss-Morris, R., Willoughby, E., Chalder, T., Johnson, M. H., \& Robinson, E. (2008). A randomized controlled trial of cognitive behavior therapy for multiple sclerosis fatigue. Psychosomatic Medicine, 70, 205-213.

Vlaeyen, J. W. S., Kole Snijders, A. M. J., Rotteveel, A. M., \& Ruesink, R. (1995). The role of fear of movement/(re)injury in pain disability. Journal of Occupational Rehabilitation, 5(4), $235-252$.

Vlaeyen, J. W. S., \& Linton, S. J. (2000). Fear-avoidance and its consequences in chronic musculoskeletal pain: A state of the art. Pain, 85(3), 317-332.

Zigmond, A. S., \& Snaith, R. P. (1983). The hospital anxiety and depression scale. Acta Psychiatrica Scandinavica, 67(6), 361370. 